(2) Open Access Full Text Article

\title{
Design, synthesis, and anti-melanogenic effects of (E)-2-benzoyl-3-(substituted phenyl)acrylonitriles
}

This article was published in the following Dove Press journal:

Drug Design, Development and Therapy

4 August 2015

Number of times this article has been viewed

\author{
Hwi Young Yun' \\ Do Hyun Kim' \\ Sujin Son' \\ Sultan Ullah' \\ Seong Jin Kim' \\ Yeon-Jeong Kim² \\ Jin-Wook Yoo' \\ Yunjin Jung' \\ Pusoon Chun ${ }^{2}$ \\ Hyung Ryong Moon' \\ 'College of Pharmacy, Pusan National \\ University, Busan, ${ }^{2}$ College of \\ Pharmacy, Inje University, Gimhae, \\ Republic of Korea
}

\begin{abstract}
Background: Tyrosinase is the most prominent target for inhibitors of hyperpigmentation because it plays a critical role in melaninogenesis. Although many tyrosinase inhibitors have been identified, from both natural and synthetic sources, there remains a considerable demand for novel tyrosinase inhibitors that are safer and more effective.

Methods: (E)-2-Benzoyl-3-(substituted phenyl)acrylonitriles (BPA analogs) with a linear $\beta$-phenyl- $\alpha, \beta$-unsaturated carbonyl scaffold were designed and synthesized as potential tyrosinase inhibitors. We evaluated their effects on cellular tyrosinase activity and melanin biosynthesis in murine B16F10 melanoma cells and their ability to inhibit mushroom tyrosinase activity.

Results: BPA analogs exhibited inhibitory activity against mushroom tyrosinase. In particular, BPA13 significantly suppressed melanin biosynthesis and inhibited cellular tyrosinase activity in B16F10 cells in a dose-dependent manner. A docking study revealed that BPA13 had higher binding affinity for tyrosinase than kojic acid.

Conclusion: BPA13, which possesses a linear $\beta$-phenyl- $\alpha, \beta$-unsaturated carbonyl scaffold, is a potential candidate skin-whitening agent and treatment for diseases associated with hyperpigmentation.
\end{abstract}

Keywords: (E)-2-benzoyl-3-(substituted phenyl)acrylonitriles, melanogenesis, tyrosinase inhibitor

\section{Introduction}

Melanins are multifunctional heterogeneous biopolymers that are widely produced by animals, plants, and microorganisms, including bacteria and fungi. ${ }^{1,2}$ Melanin pigments are classified into three main groups, ie, eumelanins and allomelanins, which are brown to black, and the yellow to reddish-brown pheomelanins. ${ }^{3-5}$ These pigments are responsible for the most striking polymorphic traits of humans and play critical roles in the camouflage and thermoregulation of animals. Moreover, eumelanin is the most important photoprotective factor and functions as a broad band ultraviolet absorbent, antioxidant, and radical scavenger in human skin. ${ }^{6-8}$ Eumelanin primarily consists of indole-type units, whereas pheomelanin is formed by the oxidative polymerization of cysteinyl conjugates of 3-(3,4-dihydroxyphenyl)L-alanine (L-DOPA). The common pathways of eumelanogenesis and pheomelanogenesis involve oxidation of L-tyrosine to L-dopaquinone, which can undergo further oxidation to dopachrome., ${ }^{3,9,10}$ On the other hand, allomelanin is synthesized by the oxidation of nitrogen-free phenols in plants and microoragnisms. ${ }^{11}$ Tyrosinase (EC 1.14.18.1), a type 3 dinuclear copper-containing metalloenzyme, exhibits the activities of monophenolase and diphenolase. It is widely distributed in nature ${ }^{12,13}$ and plays a critical role in the biosynthesis of melanin pigments. In the melanogenesis pathway, tyrosinase catalyzes steps involving the hydroxylation 
of L-tyrosine to L-DOPA, and the subsequent oxidation to the corresponding $O$-quinone. ${ }^{14,15}$ In addition, mammalian tyrosinase has been shown to catalyze a third reaction in melanogenesis, ie, the oxidation of 5,6-dihydroxyindole to indole 5,6-quinone. ${ }^{16}$

Although skin melanin is an efficient photoprotective factor, melanin hyperpigmentation in specific skin regions, such as melasma, freckles, and lentigo, can become an esthetic problem. ${ }^{17}$ Furthermore, undesirable enzymatic browning of fruits, vegetables, and some shellfish results in nutrition and quality losses. ${ }^{18,19}$ Because tyrosinase is the key regulator of melanin production, it is the most prominent target for inhibitors of hyperpigmentation. ${ }^{20,21}$

A great number of tyrosinase inhibitors, both natural and synthetic, have been identified. However, their practical uses as therapeutics and cosmetics are limited by insufficient activity or safety concerns. ${ }^{22}$ For example, hydroquinone is one of the most frequently prescribed skin-whitening agents, but it causes skin irritation and is believed to be mutagenic to mammalian cells. ${ }^{23}$ In the case of kojic acid, Japan's health ministry has ordered cosmetics companies to stop selling products containing it due to concerns about potential carcinogenicity. ${ }^{24}$ Therefore, there remains a need for novel tyrosinase inhibitors that are safe and effective. Recently we reported that the $\beta$-phenyl- $\alpha, \beta$-unsaturated carbonyl group is essential for anti-tyrosinase activity. ${ }^{25}$ Compounds I-IV (Figure 1), which contain this group, all exhibited good inhibitory activity against mushroom tyrosinase and skin-whitening effects in vivo. ${ }^{25-28}$ These compounds possess a five-membered ring, and the carbonyl and $\alpha$-carbon of the $\beta$-phenyl- $\alpha, \beta$-unsaturated carbonyl group constitute parts of this ring. Initially, an expansion of the five-membered ring to a six-membered ring, such as to barbituric acid, was attempted to increase anti-melanogenic efficacy in vitro and in vivo. Disappointingly, this ring expansion reduced tyrosinase inhibition. Alternative approaches to identify novel compounds with a potent antityrosinase effect resulted in the construction of a "linear" $\beta$-phenyl- $\alpha, \beta$-unsaturated carbonyl scaffold, not fused with a ring. In particular, the $(E)$-2-benzoyl-3-(substituted phenyl)acrylonitrile derivatives, BPA1-BPA13, bearing a linear $\alpha, \beta$-unsaturated carbonyl scaffold (Figure 2) were synthesized and their inhibitory activities against tyrosinase were examined.

\section{Materials and methods \\ Materials}

BPA1-BPA13 were synthesized in our laboratory. Mushroom tyrosinase, 3-(4-hydroxyphenyl)-L-alanine (L-tyrosine), L-DOPA, 5-hydroxy-2-hydroxymethyl-4H-4-pyranone (kojic acid), 3-(4,5-dimethyl-2-thiazolyl)-2,5-diphenyl-2Htetrazolium bromide (MTT), alpha-melanocyte-stimulating hormone ( $\alpha$-MSH), phenylmethylsulfonyl fluoride, 4-(1,1,3,3-tetramethylbutyl)phenyl-polyethylene glycol (Triton $\left.{ }^{\mathrm{TM}} \mathrm{X}-100\right)$, dimethyl sulfoxide (DMSO), ethyl alcohol, potassium dihydrogen phosphate, potassium hydrogen phosphate, benzoylacetonitrile, piperidine, sodium acetate, and several benzaldehydes were purchased from SigmaAldrich (St Louis, MO, USA). Dulbecco's Modified Eagle's Medium, fetal bovine serum, trypsin, phosphate-buffered saline, penicillin, and streptomycin were obtained from Gibco Life Technologies (Carlsbad, CA, USA). All chemicals and solvents were of analytical grade. The B16F10 mouse melanoma cell line was kindly provided by Professor Hae Young Chung (College of Pharmacy, Pusan National University, Republic of Korea).

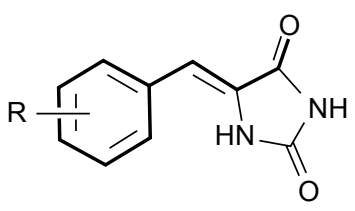

I<smiles>[R]c1ccc(C=C2SC(=O)NC2=O)cc1</smiles>

III

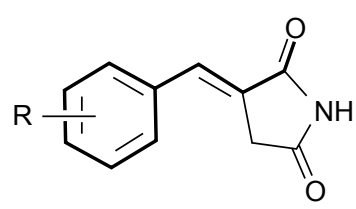

II

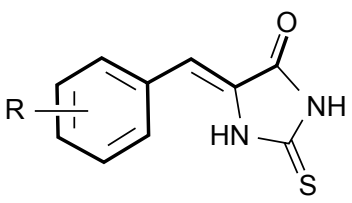

IV

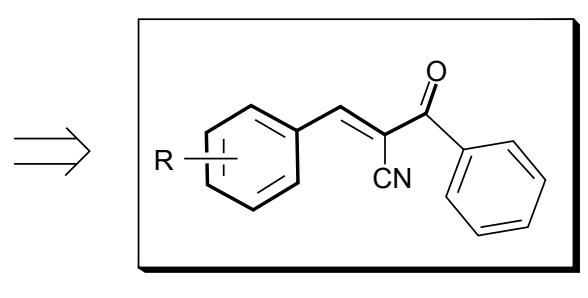

BPA1-BPA13 Anti-tyrosinase effect?

Anti-tyrosinase effect

Figure I Rationale for the design of BPA derivatives with a $\beta$-phenyl- $\alpha, \beta$-unsaturated carbonyl scaffold. Abbreviation: BPA, (E)-2-benzoyl-3-(substituted phenyl)acrylonitrile. 
<smiles>[R]c1ccc(C=C(C#N)C(=O)c2ccccc2)cc1</smiles>

\section{Benzaldehydes:}<smiles>O=Cc1ccc(O)cc1</smiles>

1<smiles>COc1ccc(C=O)cc1O</smiles>

5<smiles>O=Cc1ccccc1O</smiles>

9<smiles>O=Cc1cc(Br)c(O)c(Br)c1</smiles><smiles>O=Cc1ccc(O)cc1O</smiles>

2<smiles>COc1ccc(C=O)cc1</smiles>

6<smiles>COc1cc(C=O)cc(OC)c1OC</smiles>

10<smiles>COc1cc(C=O)ccc1O</smiles>

3<smiles>COc1ccc(C=O)cc1OC</smiles>

7<smiles>COc1cc(C=O)cc(OC)c1O</smiles>

11<smiles>CCOc1cc(C=O)ccc1O</smiles>

4<smiles>COc1ccc(C=O)c(OC)c1</smiles>

8<smiles>O=Cc1ccc(O)c(Br)c1</smiles>

12

13

Figure 2 Synthetic scheme for BPAI-BPAI3. Reagents and conditions: sodium acetate, acetic acid, reflux for BPAI-BPA9; piperidine, ethyl alcohol, reflux for BPAI0-BPAI3.

Abbreviation: BPA, (E)-2-benzoyl-3-(substituted phenyl)acrylonitrile.

\section{General procedure for synthesis of BPAI-BPA I 3}

The procedure for synthesis of BPA1-BPA13 is shown in Figure 2.

\section{Method A}

A suspension of benzoylacetonitrile (100 mg, $0.69 \mathrm{mmol})$, a benzaldehyde (1 equivalent), and sodium acetate $(169.5 \mathrm{mg}$, $2.07 \mathrm{mmol})$ in acetic acid $(0.7 \mathrm{~mL})$ was refluxed, cooled, and then water was added. The precipitates of BPA1-BPA9 generated were filtered and washed with water and organic solvents (methylene chloride and/or ethyl acetate), dissolved in methyl alcohol, and recrystallized to give the pure desired products.

\section{Method B}

A solution of benzoylacetonitrile (100 mg, $0.69 \mathrm{mmol})$ and benzaldehyde (1 equivalent) in ethanol $(3 \mathrm{~mL})$ was refluxed in the presence of piperidine $(0.02 \mathrm{~mL}, 0.20 \mathrm{mmol})$. BPA11 and BPA12 formed a precipitate on cooling, and these were filtered, then washed with water and appropriate organic solvents (methylene chloride, ethyl acetate, and/or methanol) to produce the pure desired products. BPA10 and BPA13 did not form precipitates on cooling. Thus, volatile 
substances were removed under vacuum and the resultant residues were recrystallized from methanol to obtain BPA10 and BPA13.

(E)-2-Benzoyl-3-(4-hydroxyphenyl)acrylonitrile (BPAI)

Yellow solid; reaction time, 7.5 hours; yield, $64.2 \%$; ${ }^{1} \mathrm{H}$ NMR (400 MHz, DMSO- $d_{6}$ ) $\delta 10.82$ (brs, $1 \mathrm{H}, \mathrm{OH}$ ), 7.99 (s, $1 \mathrm{H}$, vinylic H), 7.98 (d, $\left.2 \mathrm{H}, J=8.4 \mathrm{~Hz}, 2^{\prime \prime}-\mathrm{H}, 6^{\prime \prime}-\mathrm{H}\right)$, 7.76 (d, $\left.2 \mathrm{H}, J=8.4 \mathrm{~Hz}, 2^{\prime}-\mathrm{H}, 6^{\prime}-\mathrm{H}\right), 7.64$ (t, $1 \mathrm{H}, J=8.0 \mathrm{~Hz}$, $4^{\prime}-\mathrm{H}$ ), 7.53 (t, $2 \mathrm{H}, J=7.6 \mathrm{~Hz}, 3^{\prime}-\mathrm{H}, 5^{\prime}-\mathrm{H}$ ), 6.92 (d, 2 H, $J=8.4$ $\left.\mathrm{Hz}, 3^{\prime \prime}-\mathrm{H}, 5^{\prime \prime}-\mathrm{H}\right) ;{ }^{13} \mathrm{C}$ NMR (100 MHz, DMSO- $\left.d_{6}\right) \delta 190.8$, 163.7, 156.4, 136.9, 134.8, 133.5, 129.7, 129.3, 123.5, 118.3, 117.1, 106.0; LRMS (ESI-) $m / z 249$ (M) ${ }^{-}$.

(E)-2-Benzoyl-3-(2,4-dihydroxyphenyl)acrylonitrile (BPA2)

Orange-colored solid; reaction time, 8 hours; yield, $63.0 \% ;{ }^{1} \mathrm{H}$ NMR (500 MHz, DMSO- $d_{6}$ ) $\delta 10.99$ (brs, $2 \mathrm{H}, 2 \mathrm{XOH}$ ), 8.33 (s, $1 \mathrm{H}$, vinylic $\mathrm{H}), 7.84$ (d, $\left.2 \mathrm{H}, J=8.5 \mathrm{~Hz}, 2^{\prime}-\mathrm{H}, 6^{\prime}-\mathrm{H}\right), 7.69$ (d, $\left.1 \mathrm{H}, J=9.0 \mathrm{~Hz}, 6^{\prime \prime}-\mathrm{H}\right), 7.65$ (t, $\left.1 \mathrm{H}, J=8.0 \mathrm{~Hz}, 4^{\prime}-\mathrm{H}\right), 7.51$ (t, $\left.2 \mathrm{H}, J=8.0 \mathrm{~Hz}, 3^{\prime}-\mathrm{H}, 5^{\prime}-\mathrm{H}\right), 6.84$ (dd, $1 \mathrm{H}, J=8.0,2.5 \mathrm{~Hz}$, $\left.5^{\prime \prime}-\mathrm{H}\right), 6.77$ (d, $\left.1 \mathrm{H}, J=2.5 \mathrm{~Hz}, 3^{\prime \prime}-\mathrm{H}\right) ;{ }^{13} \mathrm{C}$ NMR $(100 \mathrm{MHz}$, DMSO- $\left.d_{6}\right) \delta 192.7,164.0,159.1,157.3,147.5,137.4,134.1$, 132.3, 130.0, 129.2, 121.8, 114.6, 111.4, 102.7; LRMS (ESI-) $\mathrm{m} / \mathrm{z} 265(\mathrm{M})^{-}$.

(E)-2-Benzoyl-3-(4-hydroxy-3-methoxyphenyl) acrylonitrile (BPA3)

Brownish-green solid; reaction time, 8 hours; yield, 78.4\%; ${ }^{1} \mathrm{H}$ NMR (400 MHz, DMSO- $d_{6}$ ) $\delta 10.52$ (brs, $1 \mathrm{H}, \mathrm{OH}$ ), 8.00 (s, $1 \mathrm{H}$, vinylic $\mathrm{H}), 7.77-7.75$ (m, $3 \mathrm{H}, 2^{\prime}-\mathrm{H}, 6^{\prime}-\mathrm{H}, 2^{\prime \prime}-\mathrm{H}$ ), 7.64 (t, $\left.1 \mathrm{H}, J=7.6 \mathrm{~Hz}, 4^{\prime}-\mathrm{H}\right), 7.61$ (dd, $1 \mathrm{H}, J=8.8,2.4$ $\left.\mathrm{Hz}, 6^{\prime \prime}-\mathrm{H}\right), 7.53$ (t, $\left.2 \mathrm{H}, J=8.0 \mathrm{~Hz}, 3^{\prime}-\mathrm{H}, 5^{\prime}-\mathrm{H}\right), 6.93$ (d, 1 $\left.\mathrm{H}, J=8.4 \mathrm{~Hz}, 5^{\prime \prime}-\mathrm{H}\right), 3.78\left(\mathrm{~s}, 3 \mathrm{H}, \mathrm{OCH}_{3}\right) ;{ }^{13} \mathrm{C} \mathrm{NMR}(100$ MHz, DMSO- $\left.d_{6}\right) \delta 190.8,156.6,153.5,148.5,137.0,133.5$, 129.7, 129.3, 128.0, 123.8, 118.5, 116.7, 114.9, 106.0, 56.3; LRMS (ESI-) $m / z 279$ (M)-.

\section{(E)-2-Benzoyl-3-(3-ethoxy-4-hydroxyphenyl)} acrylonitrile (BPA4)

Yellow solid; reaction time, 32 hours; yield, 95\%; ${ }^{1} \mathrm{H}$ NMR (400 MHz, DMSO- $\left.d_{6}\right) \delta 10.46$ (brs, $\left.1 \mathrm{H}, \mathrm{OH}\right), 7.98(\mathrm{~s}, 1 \mathrm{H}$, vinylic $\mathrm{H}$ ), 7.77-7.74 (m, $\left.3 \mathrm{H}, 2^{\prime}-\mathrm{H}, 6^{\prime}-\mathrm{H}, 2^{\prime \prime}-\mathrm{H}\right), 7.65$ (t, $1 \mathrm{H}, J=7.6 \mathrm{~Hz}, 4^{\prime}-\mathrm{H}$ ), 7.58 (dd, $1 \mathrm{H}, J=8.4,2.0 \mathrm{~Hz}, 6^{\prime \prime}-\mathrm{H}$ ), 7.53 (t, $\left.2 \mathrm{H}, J=7.6 \mathrm{~Hz}, 3^{\prime}-\mathrm{H}, 5^{\prime}-\mathrm{H}\right), 6.94$ (d, $1 \mathrm{H}, J=8.4 \mathrm{~Hz}$, $5^{\prime \prime}-\mathrm{H}$ ), 4.03 (q, $2 \mathrm{H}, J=7.2 \mathrm{~Hz}, \mathrm{CH}_{2} \mathrm{CH}_{3}$ ), 1.33 (t, $3 \mathrm{H}, J=7.2$ $\left.\mathrm{Hz}, \mathrm{CH}_{2} \mathrm{CH}_{3}\right) ;{ }^{13} \mathrm{C}$ NMR (100 MHz, DMSO- $\left.d_{6}\right) \delta 190.8$,
$156.7,153.7,147.7,137.0,133.5,129.7,129.3,128.1$, 123.8, 118.4, 116.8, 115.8, 106.0, 64.6, 15.2; LRMS (ESI-) $\mathrm{m} / \mathrm{z} 293(\mathrm{M})^{-}$.

\section{(E)-2-Benzoyl-3-(3-hydroxy-4-methoxyphenyl) acrylonitrile (BPA5)}

Brownish green solid; reaction time, 8 hours; yield, 74.7\%; ${ }^{1} \mathrm{H}$ NMR (500 MHz, DMSO- $d_{6}$ ) $\delta 9.66$ (s, $1 \mathrm{H}, \mathrm{OH}$ ), 7.96 (s, $1 \mathrm{H}$, vinylic $\mathrm{H}$ ), 7.79 (d, $\left.2 \mathrm{H}, J=8.5 \mathrm{~Hz}, 2^{\prime}-\mathrm{H}, 6^{\prime}-\mathrm{H}\right), 7.68$ (d, $\left.1 \mathrm{H}, J=2.5 \mathrm{~Hz}, 2^{\prime \prime}-\mathrm{H}\right), 7.67$ (t, $\left.1 \mathrm{H}, J=7.5 \mathrm{~Hz}, 4^{\prime}-\mathrm{H}\right), 7.56$ (t, $\left.2 \mathrm{H}, J=8.0 \mathrm{~Hz}, 3^{\prime}-\mathrm{H}, 5^{\prime}-\mathrm{H}\right), 7.50$ (dd, $1 \mathrm{H}, J=8.5,2.0 \mathrm{~Hz}$, $\left.6^{\prime \prime}-\mathrm{H}\right), 7.11\left(\mathrm{~d}, 1 \mathrm{H}, J=8.5 \mathrm{~Hz}, 5^{\prime \prime}-\mathrm{H}\right), 3.87\left(\mathrm{~s}, 3 \mathrm{H}, \mathrm{CH}_{3}\right) ;{ }^{13} \mathrm{C}$ NMR (100 MHz, DMSO- $\left.d_{6}\right) \delta 190.7,156.4,153.7,147.5$, 136.9, 133.6, 129.8, 129.3, 127.2, 125.2, 118.0, 116.6, 112.8, 106.9, 56.6; LRMS (ESI-) $m / z 279$ (M)-

\section{(E)-2-Benzoyl-3-(4-methoxyphenyl)acrylonitrile (BPA6)}

Yellow solid; reaction time, 8 hours; yield, $85.3 \%$; ${ }^{1} \mathrm{H}$ NMR $\left(500 \mathrm{MHz}, \mathrm{DMSO}-d_{6}\right) \delta 8.09$ (d, $2 \mathrm{H}, J=8.5 \mathrm{~Hz}, 2^{\prime \prime}-\mathrm{H}$, $\left.6^{\prime \prime}-\mathrm{H}\right), 8.08$ (s, $1 \mathrm{H}$, vinylic H), 7.81 (d, $2 \mathrm{H}, J=7.5 \mathrm{~Hz}, 2^{\prime}-\mathrm{H}$, $\left.6^{\prime}-\mathrm{H}\right), 7.68$ (td, $\left.1 \mathrm{H}, J=7.5 \mathrm{~Hz}, 4^{\prime}-\mathrm{H}\right), 7.56$ (t, $2 \mathrm{H}, J=7.5 \mathrm{~Hz}$, $\left.3^{\prime}-\mathrm{H}, 5^{\prime}-\mathrm{H}\right), 7.15$ (d, $\left.2 \mathrm{H}, J=8.5 \mathrm{~Hz}, 3^{\prime \prime}-\mathrm{H}, 5^{\prime \prime}-\mathrm{H}\right), 3.86$ (s, $\left.3 \mathrm{H}, \mathrm{CH}_{3}\right) ;{ }^{13} \mathrm{C}$ NMR $\left(100 \mathrm{MHz}\right.$, DMSO- $\left.d_{6}\right) \delta 190.7,164.3$, $156.1,136.7,134.3,133.7,129.8,129.4,125.0,118.0,115.7$, 107.4, 56.5; LRMS (ESI-) $\mathrm{m} / \mathrm{z} 263$ (M) ${ }^{-}$.

(E)-2-Benzoyl-3-(3,4-dimethoxyphenyl)acrylonitrile (BPA7)

Yellow solid; reaction time, 8 hours; yield, $78.1 \%$; ${ }^{1} \mathrm{H}$ NMR $\left(400 \mathrm{MHz}, \mathrm{DMSO}-d_{6}\right) \delta 8.05$ (s, $1 \mathrm{H}$, vinylic H), $7.78(\mathrm{~d}, 2 \mathrm{H}$, $\left.J=8.0 \mathrm{~Hz}, 2^{\prime}-\mathrm{H}, 6^{\prime}-\mathrm{H}\right), 7.76$ (s, $\left.1 \mathrm{H}, 2^{\prime \prime}-\mathrm{H}\right), 7.72$ (d, $1 \mathrm{H}, J=8.8$ $\mathrm{Hz}, 6^{\prime \prime}-\mathrm{H}$ ), 7.66 (t, $1 \mathrm{H}, J=7.2 \mathrm{~Hz}, 4^{\prime}-\mathrm{H}$ ), 7.54 (t, $2 \mathrm{H}, J=7.2$ $\left.\mathrm{Hz}, 3^{\prime}-\mathrm{H}, 5^{\prime}-\mathrm{H}\right), 7.15$ (d, $\left.1 \mathrm{H}, J=8.4 \mathrm{~Hz}, 5^{\prime \prime}-\mathrm{H}\right), 3.84$ (s, $3 \mathrm{H}$, $\mathrm{CH}_{3}$ ), 3.77 (s, $\left.3 \mathrm{H}, \mathrm{CH}_{3}\right) ;{ }^{13} \mathrm{C}$ NMR (100 MHz, DMSO- $d_{6}$ ) $\delta 190.7,156.4,154.2,149.4,136.8,133.7,129.8,129.4$, 127.5, 125.0, 118.2, 113.8, 112.6, 107.4, 56.6, 56.2; LRMS (ESI-) $\mathrm{m} / 2293$ (M)-

\section{(E)-2-Benzoyl-3-(2,4-dimethoxyphenyl)acrylonitrile} (BPA8)

Yellowish solid; reaction time, 8 hours; yield, $86.5 \%$; ${ }^{1} \mathrm{H}$ NMR (400 MHz, DMSO- $d_{6}$ ) $\delta 8.29$ (s, $1 \mathrm{H}$, vinylic H), 8.29 (d, $\left.1 \mathrm{H}, J=8.8 \mathrm{~Hz}, 6^{\prime \prime}-\mathrm{H}\right), 7.74$ (d, $\left.2 \mathrm{H}, J=7.6 \mathrm{~Hz}, 2^{\prime}-\mathrm{H}, 6^{\prime}-\mathrm{H}\right)$, 7.64 (td, $\left.1 \mathrm{H}, J=8.0,1.2 \mathrm{~Hz}, 4^{\prime}-\mathrm{H}\right), 7.53$ (t, $2 \mathrm{H}, J=7.6 \mathrm{~Hz}$, $\left.3^{\prime}-\mathrm{H}, 5^{\prime}-\mathrm{H}\right), 6.76$ (dd, $\left.1 \mathrm{H}, J=8.8,1.6 \mathrm{~Hz}, 5^{\prime \prime}-\mathrm{H}\right), 6.67$ (d, $1 \mathrm{H}$, $\left.J=1.6 \mathrm{~Hz}, 3^{\prime \prime}-\mathrm{H}\right), 3.86\left(\mathrm{~s}, 3 \mathrm{H}, \mathrm{CH}_{3}\right), 3.81\left(\mathrm{~s}, 3 \mathrm{H}, \mathrm{CH}_{3}\right) ;{ }^{13} \mathrm{C}$ NMR (100 MHz, DMSO- $\left.d_{6}\right) \delta 190.8,166.7,162.2,149.7$, 
137.1, 133.5, 130.8, 129.6, 129.3, 118.3, 113.8, 108.0, 106.4, 99.0, 57.1, 56.7; LRMS (ESI-) $m / z 293$ (M)-.

\section{(E)-2-Benzoyl-3-(2-hydroxyphenyl)acrylonitrile (BPA9)}

Beige solid; reaction time, 8 hours; yield, $94.1 \%$; ${ }^{1} \mathrm{H}$ NMR $\left(400 \mathrm{MHz}, \mathrm{DMSO}-d_{6}\right) \delta 8.40$ (s, $1 \mathrm{H}$, vinylic $\left.\mathrm{H}\right), 7.90(\mathrm{~d}, 2 \mathrm{H}$, $\left.J=7.6 \mathrm{~Hz}, 2^{\prime}-\mathrm{H}, 6^{\prime}-\mathrm{H}\right), 7.82$ (dd, $1 \mathrm{H}, J=7.6,1.2 \mathrm{~Hz}, 6^{\prime \prime}-\mathrm{H}$ ), 7.70 (td, $\left.1 \mathrm{H}, J=7.6,1.6 \mathrm{~Hz}, 4^{\prime}-\mathrm{H}\right), 7.66$ (t, $1 \mathrm{H}, J=7.6 \mathrm{~Hz}$, $\left.4^{\prime \prime}-\mathrm{H}\right), 7.51$ (t, $\left.2 \mathrm{H}, J=7.6 \mathrm{~Hz}, 3^{\prime}-\mathrm{H}, 5^{\prime}-\mathrm{H}\right), 7.46$ (d, $1 \mathrm{H}, J=8.8$ $\left.\mathrm{Hz}, 33^{\prime \prime}-\mathrm{H}\right), 7.39$ (t, $\left.1 \mathrm{H}, J=7.6 \mathrm{~Hz}, 5^{\prime \prime}-\mathrm{H}\right) ;{ }^{13} \mathrm{C}$ NMR (100 $\left.\mathrm{MHz}, \mathrm{DMSO}-d_{6}\right) \delta 192.4,158.7,154.8,146.0,136.7,134.6$, 134.3, 130.5, 130.2, 129.4, 127.0, 125.6, 118.9, 117.0; LRMS (ESI-) $m / z 249$ (M) ${ }^{-}$.

(E)-2-Benzoyl-3-(3,4,5-trimethoxyphenyl)acrylonitrile (BPAI0)

Yellow solid; reaction time, 6 days; yield, $15 \%$; ${ }^{1} \mathrm{H}$ NMR (500 MHz, DMSO- $d_{6}$ ) $\delta 8.08$ (s, $1 \mathrm{H}$, vinylic H), 7.82 (brd, $2 \mathrm{H}$, $J=7.5 \mathrm{~Hz}, 2^{\prime}-\mathrm{H}, 6^{\prime}-\mathrm{H}$ ), 7.70 (brt, $1 \mathrm{H}, J=7.5 \mathrm{~Hz}, 4^{\prime}-\mathrm{H}$ ), 7.58 (brt, $2 \mathrm{H}, J=7.5 \mathrm{~Hz}, 3^{\prime}-\mathrm{H}, 5^{\prime}-\mathrm{H}$ ), 7.52 (s, $2 \mathrm{H}, 2^{\prime \prime}-\mathrm{H}, 6^{\prime \prime}-\mathrm{H}$ ), $3.81\left(\mathrm{~s}, 6 \mathrm{H}, 2 \mathrm{XCH}_{3}\right), 3.78\left(\mathrm{~s}, 3 \mathrm{H}, \mathrm{CH}_{3}\right) ;{ }^{13} \mathrm{C} \mathrm{NMR}(100$ MHz, DMSO- $\left.d_{6}\right) \delta 190.6,156.4,153.5,142.4,136.5,133.9$, 129.9, 129.4, 127.6, 117.8, 109.5, 107.4, 61.1, 56.7; LRMS (ESI-) $m / z 323(\mathrm{M})^{-}$.

(E)-2-Benzoyl-3-(4-hydroxy-3,5-dimethoxyphenyl) acrylonitrile (BPAII)

Orange-colored solid; reaction time, 3 days; yield, 76.4\%; ${ }^{1} \mathrm{H}$ NMR (400 MHz, DMSO- $\left.d_{6}\right) \delta 8.01(\mathrm{~s}, 1 \mathrm{H}$, vinylic $\mathrm{H}$ ), 7.76 (d, $\left.2 \mathrm{H}, J=8.0 \mathrm{~Hz}, 2^{\prime}-\mathrm{H}, 6^{\prime}-\mathrm{H}\right), 7.65$ (td, $1 \mathrm{H}, J=7.2$, $\left.1.2 \mathrm{~Hz}, 4^{\prime}-\mathrm{H}\right), 7.54$ (t, $\left.2 \mathrm{H}, J=7.6 \mathrm{~Hz}, 3^{\prime}-\mathrm{H}, 5^{\prime}-\mathrm{H}\right), 7.51$ (s, $\left.2 \mathrm{H}, 2^{\prime \prime}-\mathrm{H}, 66^{\prime \prime}-\mathrm{H}\right), 3.78\left(\mathrm{~s}, 6 \mathrm{H}, 2 \mathrm{XCH}_{3}\right) ;{ }^{13} \mathrm{C} \mathrm{NMR}(100$ MHz, DMSO- $\left.d_{6}\right) \delta 190.8,156.9,148.6,142.8,137.0,133.5$, 129.7, 129.4, 122.5, 118.5, 110.2, 106.3, 56.5; LRMS (ESI-) $\mathrm{m} / \mathrm{z} 309(\mathrm{M})^{-}$.

(E)-2-Benzoyl-3-(3-bromo-4-hydroxyphenyl) acrylonitrile (BPAI2)

Yellow solid; reaction time, 6 days; yield, $84.1 \%$; ${ }^{1} \mathrm{H}$ NMR $\left(500 \mathrm{MHz}, \mathrm{DMSO}-d_{6}\right) \delta 11.67(\mathrm{~s}, 1 \mathrm{H}, \mathrm{OH}), 8.32(\mathrm{~s}, 1 \mathrm{H}$, $\left.2^{\prime \prime}-\mathrm{H}\right), 8.02$ (s, $1 \mathrm{H}$, vinylic H), 8.01 (d, $1 \mathrm{H}, J=8.0 \mathrm{~Hz}, 6^{\prime \prime}-\mathrm{H}$ ), 7.80 (d, $\left.2 \mathrm{H}, J=7.5 \mathrm{~Hz}, 2^{\prime}-\mathrm{H}, 6^{\prime}-\mathrm{H}\right), 7.68$ (td, $1 \mathrm{H}, J=7.5$, $\left.1.0 \mathrm{~Hz}, 4^{\prime}-\mathrm{H}\right), 7.56$ (t, $\left.2 \mathrm{H}, J=7.5 \mathrm{~Hz}, 3^{\prime}-\mathrm{H}, 5^{\prime}-\mathrm{H}\right), 7.12$ (d, $\left.1 \mathrm{H}, J=8.0 \mathrm{~Hz}, 5^{\prime \prime}-\mathrm{H}\right) ;{ }^{13} \mathrm{C}$ NMR $\left(100 \mathrm{MHz}, \mathrm{DMSO}-d_{6}\right)$ $\delta 190.5,159.7,154.8,137.2,136.6,133.7,133.1,129.9$, 129.4, 125.0, 117.9, 117.4, 110.9, 107.7; LRMS (ESI-) $\mathrm{m} / \mathrm{z}$ $326(\mathrm{M})^{-}$.
(E)-2-Benzoyl-3-(3,5-dibromo-4-hydroxyphenyl) acrylonitrile (BPAI3)

Orchroid solid; reaction time, 20 hours; yield, $67.8 \% ;{ }^{1} \mathrm{H}$ NMR (400 MHz, DMSO- $d_{6}$ ) $\delta 8.31$ (s, $\left.2 \mathrm{H}, 2^{\prime \prime}-\mathrm{H}, 6^{\prime \prime}-\mathrm{H}\right), 8.00$ (s, $1 \mathrm{H}$, vinylic $\mathrm{H}), 7.79$ (d, $\left.2 \mathrm{H}, J=7.2 \mathrm{~Hz}, 2^{\prime}-\mathrm{H}, 6^{\prime}-\mathrm{H}\right), 7.66$ (td, $\left.1 \mathrm{H}, J=7.6,1.2 \mathrm{~Hz}, 4^{\prime}-\mathrm{H}\right), 7.54$ (t, $2 \mathrm{H}, J=7.2 \mathrm{~Hz}, 3^{\prime}-\mathrm{H}$, $\left.5^{\prime}-\mathrm{H}\right) ;{ }^{13} \mathrm{C}$ NMR (100 MHz, DMSO- $\left.d_{6}\right) \delta 190.2,156.1,153.2$, 136.4, 135.8, 133.9, 130.0, 129.4, 126.6, 117.5, 112.6, 109.2; LRMS (ESI-) $m / z 404$ (M)-.

\section{Inhibitory effect of BPA I-BPA I 3 on mushroom tyrosinase activity}

The inhibitory effects of the synthesized compounds on mushroom tyrosinase were examined as previously described with slight modifications. ${ }^{29}$ In each well of a 96-well microplate, $10 \mu \mathrm{L}$ of each compound at different concentrations $(5,10$, or $25 \mu \mathrm{M}$ [final concentration]) was mixed with $170 \mu \mathrm{L}$ of substrate solution ( $1 \mathrm{mM} \mathrm{L-tyrosine} \mathrm{solution,} 50 \mathrm{mM}$ potassium phosphate buffer [pH 6.5], and distilled water [10:10:9, $\mathrm{v} / \mathrm{v} / \mathrm{v}])$. Mushroom tyrosinase solution $(20 \mu \mathrm{L} ; 1,000 \mathrm{U} / \mathrm{mL})$ was then added to each well, and the plate was incubated at $25^{\circ} \mathrm{C}$ for 30 minutes. The absorbances of dopachrome produced in the wells were then measured at $450 \mathrm{~nm}$ using an enzyme-linked immunosorbent assay (ELISA) reader (Tecan, Salzburg, Austria). Kojic acid was used as a reference compound. All experiments were performed in triplicate. Tyrosinase inhibition rates were calculated using:

$$
\text { Inhibition }(\%)=[1-(\mathrm{A} / \mathrm{B})] \times 100
$$

where A represents the absorbance of the test compound and $\mathrm{B}$ represents the absorbance of the blank control (no test compound).

\section{Determination of type of tyrosinase inhibition exhibited by BPAI 3}

Kinetic analysis was conducted to determine the type of inhibition exhibited by BPA13 on mushroom tyrosinase. In each well of a 96 -well plate, $10 \mu \mathrm{L}$ of $\operatorname{BPA} 13(0,5,10$, or $25 \mu \mathrm{M}$ [final concentration]) was mixed with $170 \mu \mathrm{L}$ of L-tyrosine solution $(0.5,1,2$, or $4 \mathrm{mM}$ [final concentration]), and then $20 \mu \mathrm{L}$ of mushroom tyrosinase solution $(1,000 \mathrm{U} / \mathrm{mL})$ was added. The amounts of dopachrome produced were monitored every 10 minutes for 1 hour using an ELISA reader at $450 \mathrm{~nm}$. All experiments were carried out in triplicate at a constant temperature of $25^{\circ} \mathrm{C}$. The type of inhibition was determined using LineweaverBurk plots. 


\section{In silico docking simulation of tyrosinase with BPAI 3 and kojic acid}

Of the many tools available for in silico protein-ligand docking, AutoDock vina is the most commonly used due to its automated docking capability, so was chosen for in silico protein-ligand docking simulation. The three-dimensional structure of tyrosinase obtained from the crystal structure of Agaricus bisporus (Protein Data Bank ID 2Y9X) in combination with a systematic search technique ${ }^{30,31}$ was used. The binding site of tyrosine in the crystal structure of $A$. bisporus tyrosinase was used as a docking pocket. Docking simulations were performed between tyrosinase and BPA13 or kojic acid. To prepare compounds for docking simulation, two-dimensional structures were converted into three-dimensional structures, charges were calculated, and hydrogen atoms were added using the ChemOffice program (http://www.cambridgesoft.com). LigandScout 3.1 was used to predict possible hydrogen bonding residues between compounds and tyrosinase and to identify pharmacophores.

\section{Cell culture}

B16F10 mouse melanoma cells were cultured in Dulbecco's Modified Eagle's Medium supplemented with 10\% heatinactivated fetal bovine serum and penicillin/streptomycin $(100 \mathrm{IU} / 50 \mu \mathrm{g} / \mathrm{mL})$ at $37^{\circ} \mathrm{C}$ in a humidified $5 \% \mathrm{CO}_{2}$ atmosphere.

\section{Cell viability assay}

Cell viability was assessed using an MTT assay as previously described. ${ }^{32}$ On the first day, B16F10 cells were seeded in a 24-well plate at a density of $5 \times 10^{4}$ cells/well and incubated overnight at $37^{\circ} \mathrm{C}$ in a humidified $5 \% \mathrm{CO}_{2}$ atmosphere. On the second day, cells were treated with different concentrations of BPA13 $(0,5,10$, or $25 \mu \mathrm{M})$ and incubated for 24 hours under the same conditions. On the third day, MTT solution $(0.5 \mathrm{mg} / \mathrm{mL})$ was added to each well and the plate was incubated for 2 hours at $37^{\circ} \mathrm{C}$. After removing the supernatants, the formazan crystals were dissolved in $200 \mu \mathrm{L}$ of ethyl alcohol/DMSO (1:1) and transferred to a 96-well ELISA plate. Well absorbances were measured at $570 \mathrm{~nm}$ using an ELISA reader. All experiments were performed in triplicate.

\section{Melanin content assay}

Inhibition of melanogenesis was evaluated using a melanin content assay, as previously described. ${ }^{33}$ Briefly, B16F 10 cells were seeded in a 24 -well plate $\left(5 \times 10^{4}\right.$ cells/well $)$ and incubated for 24 hours at $37^{\circ} \mathrm{C}$ in a humidified $5 \% \mathrm{CO}_{2}$ atmosphere, treated with $1 \mu \mathrm{M} \alpha-\mathrm{MSH}$ at $0,5,10$, or $25 \mu \mathrm{M}$ of BPA13 or kojic acid $(25 \mu \mathrm{M})$ for 24 hours, rinsed in phosphate-buffered saline, and lysed by incubation for 1 hour at $60^{\circ} \mathrm{C}$ in $200 \mu \mathrm{L}$ of $1 \mathrm{~N} \mathrm{NaOH}$. Lysates were transferred to a 96-well ELISA plate and absorbances were measured at $405 \mathrm{~nm}$ using an ELISA reader. Absorbances for BPA13 were expressed as percentages of kojic acid absorbance. All experiments were conducted in triplicate.

\section{Cellular tyrosinase activity assay}

Tyrosinase activity was determined by measuring the rate of L-DOPA oxidation, as previously described with minor modifications. ${ }^{34}$ Briefly, B16F10 cells were seeded in a 24-well plate at a density of $5 \times 10^{4}$ cells/well and allowed to attach overnight at $37^{\circ} \mathrm{C}$ in a humidified $5 \% \mathrm{CO}_{2}$ atmosphere. They were then treated with $1 \mu \mathrm{M} \alpha-\mathrm{MSH}$ and different concentrations $(0,5,10$, or $25 \mu \mathrm{M})$ of BPA13 or kojic acid $(25 \mu \mathrm{M})$, and incubated for 24 hours under the same conditions. After rinsing in phosphate-buffered saline, the cells were lysed with $100 \mu \mathrm{L} /$ well of lysis buffer $(90 \mu \mathrm{L}$ of $50 \mathrm{mM}$ phosphate buffer [pH 6.8], $5 \mu \mathrm{L}$ of $1 \%$ Triton X-100, and $5 \mu \mathrm{L}$ of $0.1 \mathrm{mM}$ phenylmethylsulfonyl fluoride), then frozen at $-80^{\circ} \mathrm{C}$ for 30 minutes. The lysates were thawed and centrifuged at $12,000 \mathrm{rpm}$ for 30 minutes at $4{ }^{\circ} \mathrm{C}$. The supernatants so obtained $(80 \mu \mathrm{L})$ were placed in a 96 -well ELISA, mixed with $20 \mu \mathrm{L}$ of $10 \mathrm{mM} \mathrm{L}$-DOPA, incubated for 30 minutes at $37^{\circ} \mathrm{C}$, and absorbances were measured at $500 \mathrm{~nm}$.

Tyrosinase inhibitory activity was calculated using:

$$
\text { Inhibition }(\%)=[(\mathrm{A}-\mathrm{B})-(\mathrm{C}-\mathrm{D})] /(\mathrm{A}-\mathrm{B}) \times 100
$$

where A is the absorbance of the blank (without test compound) after incubation, $\mathrm{B}$ is the absorbance of the blank (without test compound) before incubation, $\mathrm{C}$ is the absorbance of test compound after incubation, and $\mathrm{D}$ is the absorbance of test compound before incubation.

\section{Statistical analysis}

All experiments were performed in triplicate, and the results are presented as the mean \pm standard error. One-way analysis of variance followed by Tukey's test was used to determine the significances of intergroup differences. The analysis was performed using GraphPad Software (San Diego, CA, USA), and $P$-values $<0.05$ were considered to be statistically significant.

\section{Results and discussion}

BPA1-BPA9 were synthesized using a Knoevenagel reaction using a buffer system composed of sodium acetate and acetic 
acid as depicted in Figure 2. Refluxing benzoylacetonitrile and appropriate benzaldehydes (1-9) in acetic acid in the presence of sodium acetate gave $(E)$-isomers as sole products, in moderate to good yields. However, when 3,4,5-trimethoxybenzaldehyde (10), syringaldehyde (11), 3-bromo-4-hydroxybenzaldehyde (12), and 3,5-dibromo-4-hydroxybenzaldehyde (13) were used under sodium acetate/acetic acid buffered conditions, Knoevenagel condensation failed. Surprisingly, Knoevenagel condensation reactions with the same benzaldehydes in the presence of piperidine as a base catalyst in ethanol produced the desired products, ie, BPA10-BPA13. However, in most cases, a longer reaction time was needed than for sodium acetate/ acetic acid (several days versus 8 hours), although reactions also afforded $(E)$-isomers as sole products. The Knoevenagel condensation is known to primarily afford cis positioned cyano and aryl groups. The configuration of double bond was determined by comparing ${ }^{1} \mathrm{H}$ NMR spectra with those of authentic samples. ${ }^{35}$ To compare ${ }^{1} \mathrm{H}$ NMR spectra, the spectra of BPA1 and BPA6 in $\mathrm{CDCl}_{3}$ were additionally obtained. Their spectra were identical to those of authentic samples, thus confirming the presence of the $(E)$-configuration.

\section{Inhibitory effects of BPAI-BPAI3 on mushroom tyrosinase}

Of the synthesized compounds, only BPA11, BPA12, and BPA13 exhibited inhibitory activity on mushroom tyrosinase. BPA13 had a slightly greater inhibitory effect on mushroom tyrosinase than kojic acid, whereas BPA11 and BPA12 were slightly less effective (Table 1). BPA11, BPA12, and BPA13 all possess a hydroxyl group at the 4-position of the phenyl ring, and the presence of a bromo group at 3-position or at the 3-position and 5-position were found to be associated with potent anti-tyrosinase effects (see the tyrosinase inhibitory effects of BPA12 and BPA13). This result indicates that a linear $\beta$-phenyl- $\alpha, \beta$-unsaturated carbonyl scaffold is associated with potent anti-tyrosinase activity.

Table I Inhibitory effects of (E)-2-benzoyl-3-(substituted phenyl) acrylonitrile derivatives BPAI-BPAI 3 and of kojic acid on mushroom tyrosinase activity

\begin{tabular}{ll}
\hline Compound & Inhibition (\%) \\
\hline BPAI-BPAI0 & $\mathrm{NI}$ \\
BPAII & $22.53 \pm 1.44$ \\
BPAI2 & $30.13 \pm 2.06$ \\
BPAI3 & $44.68 \pm 0.62$ \\
Kojic acid & $39.35 \pm 0.83$ \\
\hline
\end{tabular}

Notes: Mean \pm standard error are shown. The results are representative of at least three independent experiments, which were conducted at a concentration of $25 \mu \mathrm{M}$.

Abbreviation: $\mathrm{NI}$, no inhibition.

\section{Determining type of tyrosinase inhibition}

The mode of the inhibitory action of BPA13 on mushroom tyrosinase was determined based on the effects of BPA13 on $\mathrm{Km}$ (dissociation constant) and $\mathrm{V}_{\max }$ (maximum reaction velocity) values using a Lineweaver-Burk plot. As shown in Figure 3, inhibition of mushroom tyrosinase resulted in a double-reciprocal plot, on which the four straight lines with different slopes crossed the y-axis at similar points. This result showed $\mathrm{V}_{\max }$ values were not affected by BPA13 concentration. On the other hand, Km values increased gradually as the concentration of BPA13 was increased. These observations show that BPA13 competitively and dose-dependently inhibits mushroom tyrosinase.

\section{In silico docking simulation of tyrosinase and BPAI3}

A docking study was performed to explore whether BPA13 directly inhibits tyrosinase by binding to its active site. The docking simulation was successful (Figure 4). The binding energy of BPA13 was found to be $-7.7 \mathrm{kcal} / \mathrm{mol}$ (AutoDock vina) and that of kojic acid was $-5.4 \mathrm{kcal} / \mathrm{mol}$. Ligand-toreceptor docking scores represent different energies, such as electrostatic energy, van der Waals energy, and solvation energy. Docking simulation indicated that binding between BPA13 and tyrosinase is more energetically favored than kojic acid-to-tyrosinase binding. We searched for binding residues of tyrosinase interacting with BPA13 using LigandScout 3.1. According to the results obtained, His85 and Glu322 of tyrosinase are mainly responsible for hydrogen bonding interactions with the phenolic hydroxyl of BPA13 (Figure 4B). In addition, the phenyl ring of the benzoyl group of BPA13

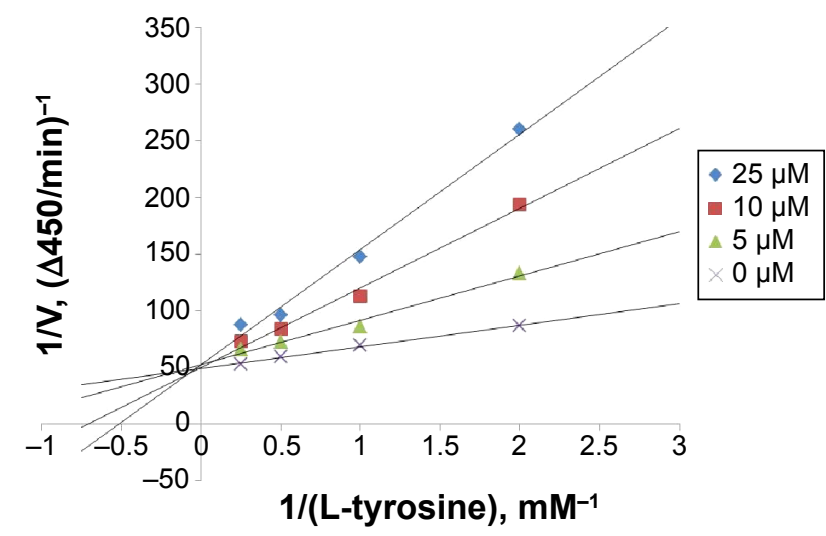

Figure 3 Determination of the nature of tyrosinase inhibition. Inhibition type was determined using Lineweaver-Burk plots. Results are mean values of $I / V$, the inverse of the increase in the absorbance per minute at the different L-tyrosine concentrations. A modified Michaelis-Menten equation was utilized: $1 / \mathrm{V}_{\max }=\mathrm{I} /$ $\mathrm{Km}(\mathrm{I}+[\mathrm{S}] / \mathrm{Ki}) . V$ denotes the velocity of the reaction, $\mathrm{S}$ the L-tyrosine concentration, $\mathrm{Km}$ the dissociation constant, and $\mathrm{Ki}$ the inhibitor constant. 
A

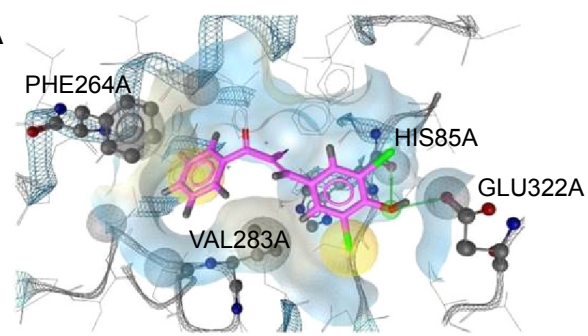

C

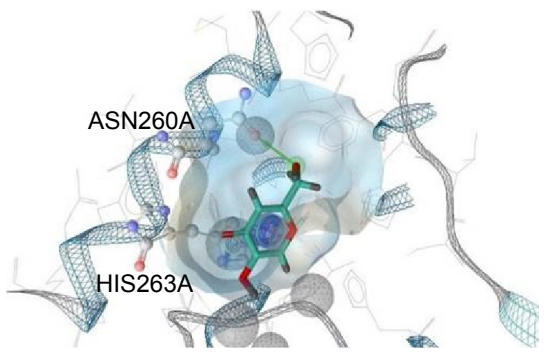

B PHE264A

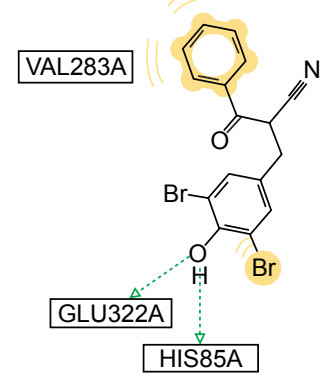

D $\frac{A S N 260 A}{\nabla}$<smiles>COCc1cc(=O)c(O)co1</smiles>

\begin{tabular}{|ll|}
\hline Compounds & $\begin{array}{l}\text { Tyrosinase } \\
\text { docking score }\end{array}$ \\
\hline BPA13 & $-7.7 \mathrm{kcal} / \mathrm{mol}$ \\
\hline Kojic acid & $-5.4 \mathrm{kcal} / \mathrm{mol}$ \\
\hline
\end{tabular}

HIS263A

Figure 4 Docking simulation between tyrosinase and BPAI 3 or kojic acid.

Notes: (A) Docking simulation between mushroom tyrosinase and BPAI3 shown in magenta. (B) The pharmacophore model generated using the LigandScout 3.1 program indicated possible hydrogen bonding interactions and hydrophobic interactions between tyrosinase residues and BPAI3. (C) Docking simulation between tyrosinase and kojic acid, used as a positive control. (D) The pharmacophore model generated using the LigandScout 3.I program indicated a possible hydrogen bonding interaction between tyrosinase residues and kojic acid.

Abbreviation: BPA, (E)-2-benzoyl-3-(substituted phenyl)acrylonitrile.

was found to participate in hydrophobic interactions with two amino acid residues of tyrosinase, Phe264 and Val283. On the other hand, kojic acid had one hydrogen bonding interaction with Asn260 of tyrosinase (Figure 4D). In order to identify other tyrosinase residues important for inhibitor binding, we examined common tyrosinase residues located within $3 \AA$ of BPA13 and kojic acid. We found three amino acid residues, Asn260, His263, and Val283. Therefore, it seems that these residues contribute importantly to the inhibition of tyrosinase activity.

\section{Cell viability assay}

The effect of BPA13 on B16F10 cell viability was evaluated using an MTT assay (Figure 5). After 24 hours of exposure to 5,10 , or $25 \mu \mathrm{M}$ of BPA13, cell viabilities were $100.5 \%$, $99.0 \%$, and $91.1 \%$ relative to untreated controls, which implies that BPA13 is non-cytotoxic at concentrations of $\leq 25 \mu \mathrm{M}$. $^{36}$

\section{Melanin content assay}

The inhibitory effect of BPA13 on $\alpha$-MSH-stimulated melanogenesis in B16F10 cells was assessed by measuring intracellular melanin. Melanin contents were substantially lower in $\alpha-\mathrm{MSH} / \mathrm{BPA} 13$ co-treated cells than in cells treated with $\alpha$-MSH alone. Furthermore, at 0-25 $\mu \mathrm{M}$ BPA13 dosedependently inhibited melanin synthesis, and at $25 \mu \mathrm{M}$ BPA13 was as effective as kojic acid (Figure 6).

\section{Cellular tyrosinase activity assay}

The effect of BPA13 on cellular tyrosinase activity was examined in $\alpha-\mathrm{MSH}$-treated B16F10 cells. BPA13 significantly reduced tyrosinase activity in a dose-dependent manner (Figure 7). This result implies that the inhibitory

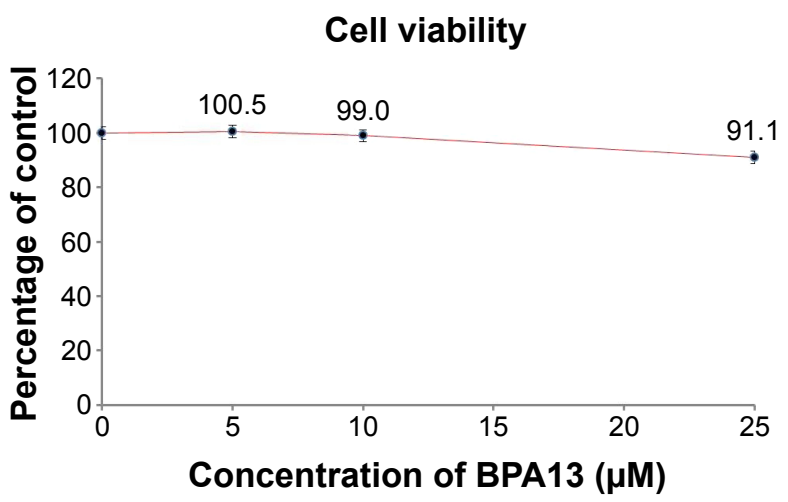

Figure 5 The effect of BPAI 3 on the viability of BI6FIO cells. Cells were treated with different concentrations of BPAI 3 for 24 hours. Viabilities are expressed as mean values of the percentage relative to the untreated control cells. Results are representative of at least three independent experiments.

Abbreviation: BPA, (E)-2-benzoyl-3-(substituted phenyl)acrylonitrile. 


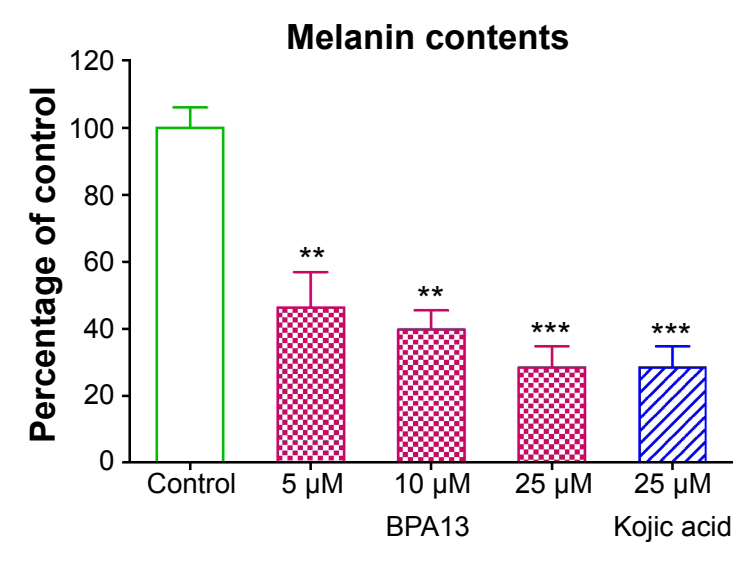

Figure 6 The effect of BPAI 3 on $\alpha$-MSH-mediated melanogenesis. BI6FIO cells were co-treated with $\alpha-M S H$ and BPAI 3 (at different concentrations) or kojic acid for 24 hours. Melanin contents are expressed as mean values of percentages versus control cells treated with $\alpha-M S H$ alone. The asterisk denotes statistical significance between control cells and the cells exposed to BPAI 3 or kojic acid. ${ }^{* * P}<0.0 \mathrm{I}$; $* * * P<0.001$. Results are representative of at least three independent experiments. Abbreviation: BPA, (E)-2-benzoyl-3-(substituted phenyl)acrylonitrile.

effect of BPA13 on melanin biosynthesis is due to its ability to suppress tyrosinase activity.

\section{Conclusion}

In this study, we designed and synthesized BPA analogs with a linear $\beta$-phenyl- $\alpha, \beta$-unsaturated carbonyl scaffold and evaluated their inhibitory effects on tyrosinase activity and melanogenesis. The synthesized compounds BPA11, BPA11, and BPA13 exhibited an inhibitory activity against mushroom tyrosinase, and BPA13 significantly and dosedependently suppressed melanin biosynthesis. At $25 \mu \mathrm{M}$, BPA13 was as effective as kojic acid at reducing melanin production and at this concentration had no cytotoxic effect.

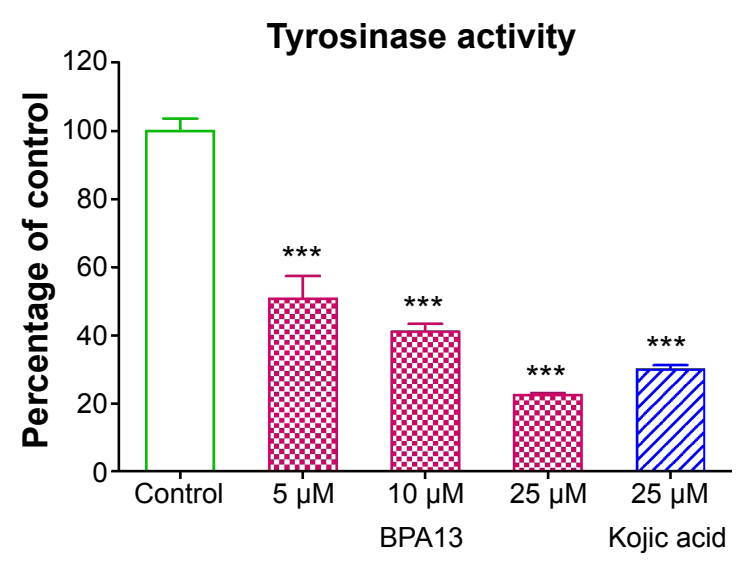

Figure 7 The effect of BPAI3 on cellular tyrosinase activity. BI6FIO cells were co-treated with $\alpha-M S H$ and BPAI 3 (at different concentrations) or kojic acid for 24 hours. Tyrosinase activities are expressed as mean values of percentages versus control cells treated with $\alpha-\mathrm{MSH}$ alone. The asterisk denotes statistical significance between control cells and cells treated with BPAI 3 or kojic acid: ${ }^{*} P<<0.0$; $* * * P<0.001$. Results are representative of at least three independent experiments. Abbreviation: BPA, (E)-2-benzoyl-3-(substituted phenyl)acrylonitrile.
Our docking simulation study revealed that BPA13 is able to bind directly to the active site of mushroom tyrosinase with higher binding affinity than kojic acid, and our kinetic study showed that BPA13 competitively inhibits tyrosinase. Collectively, BPA13 appears to be a promising treatment for diseases associated with hyperpigmentation and to be a possible skin-whitening agent.

\section{Acknowledgments}

This work was supported by the Basic Science Research Program through the National Research Foundation of Korea funded by the Korean Ministry of Education, Science and Technology (NRF-2013R1A1A2009949) and by a National Research Foundation of Korea grant funded by the Korean government (2009-008358).

\section{Disclosure}

The authors have no conflict of interest to declare.

\section{References}

1. della-Cioppa G, Garger SJ, Sverlow GG, Turpen TH, Grill LK. Melanin production in Escherichia coli from a cloned tyrosinase gene. Biotechnology (N Y). 1990;8(7):634-638.

2. Carletti G, Nervo G, Cattivelli L. Flavonoids and melanins: a common strategy across two kingdoms. Int J Biol Sci. 2014;10(10):1159-1170.

3. Slominski A, Tobin DJ, Shibahara S, Wortsman J. Melanin pigmentation in mammalian skin and its hormonal regulation. Physiol Rev. 2004; 84(4):1155-1228.

4. Mapunya MB, Nikolova RV, Lall N. Melanogenesis and antityrosinase activity of selected South African plants. Evid Based Complement Alternat Med. 2012;2012:374017.

5. d'Ischia M, Wakamatsu K, Napolitano A, et al. Melanins and melanogenesis: methods, standards, protocols. Pigment Cell Melanoma Res. 2013; 26(5):616-633.

6. Rózanowska M, Sarna T, Land EJ, Truscott TG. Free radical scavenging properties of melanin interaction of eu- and pheo-melanin models with reducing and oxidising radicals. Free Radic Biol Med. 1999; 26(5-6):518-525.

7. Sturm RA, Teasdale RD, Box NF. Human pigmentation genes: identification, structure and consequences of polymorphic variation. Gene. 2001;277(1-2):49-62.

8. Lin JY, Fisher DE. Melanocyte biology and skin pigmentation. Nature. 2007;445(7130):843-850.

9. Wakamatsu K, Ito S. Advanced chemical methods in melanin determination. Pigment Cell Res. 2002;15(3):174-183.

10. Hearing VJ. Determination of melanin synthetic pathways. J Invest Dermatol. 2011;131(E1):E8-E11.

11. Plonka PM, Grabacka M. Melanin synthesis in microorganisms biotechnological and medical aspects. Acta Biochim Pol. 2006;53(3): 429-443.

12. van Gelder CW, Flurkey WH, Wichers HJ. Sequence and structural features of plant and fungal tyrosinases. Phytochemistry. 1997;45(7): $1309-1323$.

13. Fairhead M, Thöny-Meyer L. Bacterial tyrosinases: old enzymes with new relevance to biotechnology. N Biotechnol. 2012;29(2):183-191.

14. Kobayashi T, Vieira WD, Potterf B, Sakai C, Imokawa G, Hearing VJ. Modulation of melanogenic protein expression during the switch from eu- to pheomelanogenesis. J Cell Sci. 1995;108 Pt 6:2301-2309. 
15. Chang TS. An updated review of tyrosinase inhibitors. Int J Mol Sci. 2009;10(6):2440-2475.

16. Körner A, Pawelek J. Mammalian tyrosinase catalyzes three reactions in the biosynthesis of melanin. Science. 1982;217(4565):1163-1165.

17. Im S, Kim J, On WY, Kang WH. Increased expression of alphamelanocyte-stimulating hormone in the lesional skin of melasma. Br J Dermatol. 2002;146(1):165-167.

18. Kubo I, Kinst-Hori I, Kubo Y, Yamagiwa Y, Kamikawa T, Haraguchi H. Molecular design of antibrowning agents. J Agric Food Chem. 2000; 48(4):1393-1399.

19. Coates CJ, Nairn J. Hemocyanin-derived phenoloxidase activity: a contributing factor to hyperpigmentation in Nephrops norvegicus. Food Chem. 2013;140(1-2):361-369.

20. Kim YJ, Uyama H. Tyrosinase inhibitors from natural and synthetic sources: structure, inhibition mechanism and perspective for the future. Cell Mol Life Sci. 2005;62(15):1707-1723.

21. Solano F, Briganti S, Picardo M, Ghanem G. Hypopigmenting agents: an updated review on biological, chemical and clinical aspects. Pigment Cell Res. 2006;19(6):550-571.

22. Curto EV, Kwong C, Hermersdörfer $\mathrm{H}$, et al. Inhibitors of mammalian melanocyte tyrosinase: in vitro comparisons of alkyl esters of gentisic acid with other putative inhibitors. Biochem Pharmacol. 1999; 57(6):663-672.

23. Parvez S, Kang M, Chung HS, et al. Survey and mechanism of skin depigmenting and lightening agents. Phytother Res. 2006;20(11):921-934.

24. Fuyuno I. Spotlight turns on cosmetics for Asian skin. Nature. 2004; 432(7020):938.

25. Kim HR, Lee HJ, Choi YJ, et al. Benzylidene-linked thiohydantoin derivatives as inhibitors of tyrosinase and melanogenesis: importance of the $\beta$-phenyl- $\alpha, \beta$-unsaturated carbonyl functionality. Med Chem Comm. 2014;5(9):1410-1417.

26. Ha YM, Kim J-A, Park YJ, et al. Analogs of 5-(substituted benzylidene) hydantoin as inhibitors of tyrosinase and melanin formation. Biochim Biophys Acta. 2011;1810(6):612-619.
27. Ha YM, Kim J-A, Park YJ, et al. Synthesis and biological activity of hydroxybenzylidenyl pyrrolidine-2,5-dione derivatives as new potent inhibitors of tyrosinase. Med Chem Comm. 2011;2(6):542-549.

28. Kim SH, Ha YM, Moon KM, et al. Anti-melanogenic effect of (Z)-5-2, 4-dihydroxybenzylidene)thiazolidine-2,4-dione, a novel tyrosinase inhibitor. Arch Pharm Res. 2013;36(10):1189-1197.

29. Hyun SK, Lee WH, Jeong da M, Kim Y, Choi JS. Inhibitory effects of kurarinol, kuraridinol, and trifolirhizin from Sophora flavescens on tyrosinase and melanin synthesis. Biol Pharm Bull. 2008;31(1):154-158.

30. Moustakas DT, Lang PT, Pegg S, et al. Development and validation of a modular, extensible docking program: DOCK 5. J Comput Aided Mol Des. 2006;20(10/11):601-619.

31. Morris GM, Goodsell DS, Halliday RS, et al. Automated docking using a Lamarckian genetic algorithm and empirical binding free energy function. J Comput Chem. 1998;19(14):1639-1662.

32. Riss T, Moravec RA, Niles AL, Benink HA, Worzella TJ, Minor L. Cell viability assays. In: Assay Guidance Manual. Bethesda, MD, USA: Eli Lilly \& Company and the National Center for Advancing Translational Sciences; 2013.

33. Chen LG, Chang WL, Lee CJ, Lee LT, Shih CM, Wang CC. Melanogenesis inhibition by gallotannins from Chinese galls in B16 mouse melanoma cells. Biol Pharm Bull. 2009;32(8):1447-1452.

34. Bae SJ, Ha YM, Kim JA, et al. A novel synthesized tyrosinase inhibitor: (E)-2-((2,4-dihydroxyphenyl)diazenyl)phenyl 4-methylbenzenesulfonate as an azo-resveratrol analog. Biosci Biotechnol Biochem. 2013;77(1):65-72.

35. Deshpande SJ, Leger PR, Sieck SR. Microwave synthesis of $\alpha$-cyano chalcones. Tetrahedron Lett. 2012;53(14):1772-1775.

36. Kong N, Jiang T, Zhou Z, Fu J. Cytotoxicity of polymerized resin cements on human dental pulp cells in vitro. Dent Mater. 2009;25(11): $1371-1375$.
Drug Design, Development and Therapy

\section{Publish your work in this journal}

Drug Design, Development and Therapy is an international, peerreviewed open-access journal that spans the spectrum of drug design and development through to clinical applications. Clinical outcomes, patient safety, and programs for the development and effective, safe, and sustained use of medicines are a feature of the journal, which

\section{Dovepress}

has also been accepted for indexing on PubMed Central. The manuscript management system is completely online and includes a very quick and fair peer-review system, which is all easy to use. Visit http://www.dovepress.com/testimonials.php to read real quotes from published authors. 